Math. Z. 227, 245-262 (1998)

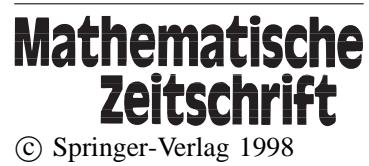

\title{
On affine actions of Lie groups
}

\author{
Abdelghani Zeghib \\ UMPA, ENS Lyon, 46, allée d'Italie, F-69364 Lyon cedex 7, France \\ (e-mail: Zeghib@umpa.ens-lyon.fr)
}

Received 2 August 1995; in final form 2 December 1996

Smooth actions of certain Lie groups are (vaguely) conjectured to be "rigid". In considering such conjectures it is natural to start by studying actions that are already rigid. Indeed from these conjectures themselves one might see that this is not so restrictive, and, on the other hand, this may help to understand the general case.

Among existing work in this field, the articles of Zimmer ([Zim1], [Zim2]...) and Gromov ([Gro], [D-G]) are fundamental. Their approaches are respectively ergodic theoretical and "analytical". In this paper, we obtain, by elementary geometrical and "algebraic" methods some new results and new proofs of existing results and (partial) answers to some (partial) questions.

Rigidity questions concern in some sense the transverse structure of the underlying "foliation", but here we deal with questions about the geometry and topology of leaves. In fact, a fundamental question is, do we actually have a

foliation ? Equivalently: what conditions ensure that the action is everywhere locally free?

Our results concern essentially actions of semi-simple Lie groups preserving a pseudo-riemannian or an unimodular affine structure on a compact manifold. The principal ones are Theorems 3.1, 3.2, 4.7, 4.8, 4.9, 5.2, 7.5, 8.5 and 8.9.

We will always assume the actions locally faithful. All semi-simple groups that we consider here are linear, without compact factor.

Let us begin by recalling examples (from [D-G]) of actions of semi-simple Lie groups.

\section{Examples}

\subsection{Linear actions}

Let $\rho: G \rightarrow G L(n, \mathbf{R})$ be a representation of a Lie group $G$. Given a real $\lambda>0$, let $\mathbf{H}_{n}$ be the Hopf manifold, quotient of $\mathbf{R}^{n}-\{0\}$ by $x \rightarrow \lambda x$. It is diffeomorphic 
to $S^{1} \times S^{n-1}$ equipped with an affine flat connection. The group $G$ acts on $\mathbf{H}_{n}$ by affine transformations (in the case of $G=S L(n, \mathbf{R})$ the action is in fact transitive). However this action has no invariant measures (notice nevertheless that there is a kind of invariant conformal measure...).

To get examples with more trivial topology one may let $\rho(G)$ act projectively on the sphere $S^{n-1}$. Such an action preserves neither a measure nor a connection.

\subsection{Homogeneous actions}

Let $I$ be a Lie group, $G$ a (closed) Lie subgroup of $I, \Gamma$ a discrete subgroup of $I$ and let $M$ be the quotient $I / \Gamma$, that we suppose to be compact. Then $G$ acts by multiplication on the left on $M$.

\subsubsection{Canonical and warped connections}

The Lie group $I$ has a canonical (torsion free) bi-invariant connection that is given, for right invariant vector fields $X$ and $Y$ by: $\nabla_{X} Y=(1 / 2)[Y, X]$. It passes to an $I$-invariant connection on the quotient $M=I / \Gamma$ (It is here that we require $\Gamma$ to be discrete).

Any $I$-right invariant connection on $I$ (that is a connection with constant Christoffel symbols in a right invariant frame field) descends to a connection on $M$. Such a (warped) connection $\nabla^{W}$ is given by: $\nabla_{X}^{W} Y=(1 / 2)[Y, X]+W(X, Y)$, where $W: \mathscr{T} \times \mathscr{T} \rightarrow \mathscr{T}$ is a symmetric bilinear form, where $\mathscr{T}$ is the Lie algebra of $I$.

Moreover if $G$ is a subgroup of $I$, then $\nabla^{W}$ is invariant under the left action of $G$ if and only if $W$ is $\operatorname{Ad}(G)$ equivariant. In this case $\nabla^{W}$ gives a connection on $I / \Gamma$ which is invariant under the left action of $G$.

One needs unimodularity conditions to get actions which are in addition volume preserving.

\subsubsection{Pseudo-riemannian metrics}

Some Lie groups admit bi-invariant pseudo-riemannian metrics. This is indeed the case of semi-simple Lie groups, for which the Killing form provides such a structure, and also some solvable groups [M-R]. In general metrics that pass to the quotients $I / \Gamma$ are given by (scalar) bilinear forms: $b: \mathscr{T} \times \mathscr{T} \rightarrow \mathbf{R}$. As above they are $G$-invariant, if and only if, $b$ is $\operatorname{Ad}(G)$-invariant.

Such $G$-actions are in particular connection and volume preserving.

\subsubsection{Locally homogeneous case}

Now let $J$ be a connected closed Lie subgroup of $I$. Instead of taking quotients of $I$ as above, one may consider the homogeneous space $J \backslash I$, and its quotients 
$M=J \backslash I / \Gamma$ (if they exist as a compact Hausdorff manifold). The Normalizer $\operatorname{Nor}_{I}(J)$ acts by the left on $M$. Similar conditions as above, for structures on $J \backslash I$, ensure that they pass to $G$-invariant structures on $M$, where $G$ is a subgroup of $\operatorname{Nor}_{I}(J)$.

For example if both $I$ and $J$ are semi-simple, the Killing form allows one to construct pseudo-riemannian metrics satisfying all the invariance conditions.

\subsection{Suspensions}

Let $\Gamma$ be a co-compact lattice of $G$ acting on a manifold $F$. Consider the suspension manifold $M=G \times F /(g, f) \sim\left(g \gamma, h_{\gamma}(f)\right)$, where for $\gamma \in \Gamma, h_{\gamma}$ denotes the associated homeomorphism of $F$. The group $G$ acts on the left on $M$. All the orbits are covering of $G / \Gamma$ and so in particular the action is everywhere locally free. It is volume preserving (resp. connection preserving) if the action of $\Gamma$ is.

\subsection{Foliated actions}

We first give a special case of the construction. Let $\mathscr{D}$ be a 2-dimensional lamination. Assume it hyperbolic, that is there is a leafwise riemannian metric such that the curvature of the leaves is identically -1 . Then each leaf $L$ is a quotient $\mathbf{H}^{2} / \Gamma$ of the 2 hyperbolic disc, and hence $G=\operatorname{PSL}(2, \mathbf{R})$ acts (by the left) on the unitary tangent bundle $T^{1} L=T^{1} \mathbf{H}^{2} / \Gamma=P S L(2, \mathbf{R}) / \Gamma$. This also extends in a canonical way to an action of $\operatorname{PSL}(2, \mathbf{R})$ on the unit tangent bundle of the lamination $T^{1} \mathscr{B}$. One way to see that this is canonical, is to recall the following geometric interpretation of the $\operatorname{PSL}(2, \mathbf{R})$ action on $\mathbf{H}^{2}$ : the orbits of a one parameter group are exactly curves with fixed geodesic curvature.

The general construction is the following. Let $\mathscr{D}$ be a lamination of any dimension with leaves endowed with a $(G, X)$ geometric structure. So in particular, $G$ acts transitively on the model space $X$, with isotropy group $K$, say. We assume $X$ simply connected and that the geometric structure is complete on each leaf, that is each leaf $L$ is covered by $X$. Consider the space $\mathrm{M}$ of (global) isometric leafwise immersions of $X$ into the leaves of $\mathscr{B}$. It fibers over the support of $\mathscr{B}$ with fiber type $K$, and it is endowed with an action, by composition at the source, of $G$.

\section{Geometry of Killing fields of pseudo-riemannian metrics}

\subsection{Killing fields}

Let $(M,<,>)$ be a pseudo-riemannian manifold, and $\nabla$ its associated Levi-Civita connection. Recall the following $[\mathrm{K}-\mathrm{N}]$ :

Fact 2.1 A vector field $X$ in $M$ is a Killing field (that is, its flow preserves $<,>$ ) if and only if for any $x \in M$, the linear map $D_{x} X: u \rightarrow \nabla_{u} X$ is antisymmetric with respect to $\langle$,$\rangle , i.e. \left\langle\nabla_{u} X, v\right\rangle+\left\langle u, \nabla_{v} X\right\rangle=0$. 
Proposition 2.2 Consider an isometric action of the affine group of the line $A G$, on a finite volume pseudo-riemannian manifold $M$, that is given by two Killing fields $X$ and $Y$ satisfying $[X, Y]=-Y$. Then, $Y$ is isotropic and its orbits are (affinely parameterized) geodesics.

Proof. Let $\phi^{t}$ be the flow of $X$. Then $\phi^{t} Y=\exp (-t) Y$. Therefore $<\phi^{t} Y(x), \phi^{t} Y(x)>=\exp (-2 t)<Y\left(\phi^{t} x\right), Y\left(\phi^{t} x\right)>$. In particular the function $x \rightarrow<Y(x), Y(x)>$ is decreasing along the $\phi$-orbits. Hence it is identically 0 , because $\phi^{t}$ preserves the finite pseudo-riemannian volume. Therefore $Y$ is isotropic. To prove that it has geodesic orbits, we apply:

Lemma 2.3 A Killing field $Y$ on a pseudo-riemannian manifold with constant norm $\langle Y, Y\rangle$, has geodesic orbits: $\nabla_{Y} Y=0$. More generally, if $f=\langle Y, Y\rangle$, then gradf $=-(1 / 2) \nabla_{Y} Y$. Thus $Y$ has geodesic orbits, if and only if it has a constant length.

Proof. From the fact above: $\left\langle\nabla_{Y} Y, u>+<\nabla_{u} Y, Y>=0\right.$. Thus $<\nabla_{Y} Y, u>=-(1 / 2) u .<Y, Y>$.

Remark 2.4 When we merely have an invariant affine connection instead of a pseudo-riemannian metric, the proposition (that is, the orbits of $Y$ are geodesic) above is false. Examples may be constructed from 1.2.1 (see also 7.4).

\subsection{Killing Lie algebra}

Here we generalize the lemma above to actions of Lie groups. Everywhere in this article, the Lie algebra of a Lie group $G$ is denoted by $\mathscr{G}$.

Theorem 2.5 Let $G$ be a Lie group acting isometrically on a pseudo-riemannian manifold $(M,<,>)$. Suppose that for any $X \in \mathscr{G}$, the length $\langle X(x), X(x)\rangle$ is constant in $x$. (This is for example the case if all the orbits of $G$ are isotropic). Then (all) the orbits of $G$ are geodesic. Moreover, the induced connection on the orbits comes from the canonical one on $G$. That is, for any $x \in M, X, Y \in \mathscr{G}$, we have: $\nabla_{X} Y(x)=(1 / 2)[X, Y](x)$.

Proof. Let $x \in M$. Any tangent vector $u$ to $G x$ at $x$ coincides with the evaluation at $x$ of some Killing field $X \in \mathscr{G}$. From the lemma above the orbits of $X$ are geodesic. Hence the geodesic determined by $u$ is contained in $G x$. Therefore, since $u$ is arbitrary, $G x$ is geodesic.

For the formula, let $X$ and $Y$ be elements of $\mathscr{G}$ and $U$ any vector field on $M$. Since $D Y$ and $D X$ are antisymmetric:

$$
\begin{aligned}
& <\nabla_{X} Y, U>+<X, \nabla_{U} Y>=0, \\
& <\nabla_{Y} X, U>+<Y, \nabla_{U} X>=0 .
\end{aligned}
$$

Remember that $\langle X, Y\rangle$ is constant (since $\langle X, X\rangle,\langle Y, Y\rangle$ and $\langle X+Y, X+Y\rangle$ are so) and hence $\left\langle X, \nabla_{U} Y\right\rangle=-\left\langle Y, \nabla_{U} X\right\rangle$. Thus $<\nabla_{X} Y+\nabla_{Y} X, U>=0$. Since this is true for any $U$, we get: $\nabla_{X} Y+\nabla_{Y} X=0$. Adding this to $\nabla_{X} Y-\nabla_{Y} X=[X, Y]$, we obtain the desired formula. 
Corollary 2.6 If a Lie group acts isometrically on $(M,<,>)$, with isotropic orbits, then the stabilizer algebra of a point of $M$ is an ideal of $\mathscr{G}$.

\section{Actions of semi-simple Lie groups}

From the last corollary we deduce the following result which allows us to "completely understand" the isotropic case, when $G$ is semi-simple.

Theorem 3.1 Let $G$ be a semi-simple Lie group acting isometrically on a pseudoriemannian manifold $(M,<,>)$, with all of its orbits isotropic. Then $G$ acts everywhere locally freely, with geodesic orbits. The induced connection on the orbits is the projection of the canonical one on the group $G$.

Proof. We can assume that $G$ is simply connected and so splits into simple factors. From the Corollary, if the action of $G$ is not locally free, then the same is true for some factor. Therefore we may suppose $G$ to be simple. The Corollary says in this case that the stabilizer algebra of any point is either $\{0\}$ or $\mathscr{G}$. The last case corresponds to $G$-fixed points. Suppose $x$ is such a point. Then we have a derivative representation $\rho: G \rightarrow G L\left(T_{x} M\right.$ ) (in fact $\rho$ takes values in the orthogonal group of $\left.\left(T_{x} M,<,>\right)\right)$. Now, since $G$ preserves a connection, its action is linearized near $x$, via the exponential map $\exp _{x}$. Thus the Corollary statement translates to: the stabilizer in $\rho(G)$ of a vector $u \in T_{x} M$ is either trivial or $\rho(G)$ itself. By basic facts from the theory of representation of simple Lie groups, this implies $\rho$ is trivial. The connection preserving property implies thus that $G$ acts trivially on $M$, but we have assumed it acting faithfully!

Another situation that we understand well is described in the following:

Theorem 3.2 Let $G$ be a semi-simple Lie group with no compact factor acting isometrically on a finite volume pseudo-riemannian manifold $(M,<,>)$. Suppose that the action of $G$ is ergodic. Then $G$ acts everywhere locally freely, with geodesic orbits. The induced metric (resp. the connection) on the orbits is the projection of a bi-invariant metric (resp. the canonical connection) on the group $G$.

Proof. If a semi-simple Lie group $G$ acts ergodically, then most (a non empty open set) of its one parameter groups act so. Indeed the real content of Moore's ergodic Theorem is that, if $G$ acts ergodically then all non compact one parameter groups of $G$ act ergodically (they are in fact mixing). Such a one parameter group leaves invariant the length function $\langle X, X\rangle$ of its infinitesimal generator $X$, and hence this function is constant.

Therefore, in an open subset, the length function is constant. This extends by bilinearity, to all the elements of $\mathscr{G}$. Then we apply 2.3 .

To see that the metrics along the orbits come from a bi-invariant metric on the group, observe that their pull backs to $G$ are $G$-left invariant metrics on $G$. The Killing fields on the orbits, corresponds to right invariant vector fields (that 
act by the left) on $G$. The constancy of their lengths means exactly that the metric is $G$-right invariant.

\section{Actions of simple split Lie groups}

In the case where we do not have dynamical information, such as ergodicity, or a geometrical one, such as isotropy, we have the following general fact:

Corollary 4.1 (of 2.2) Let $G$ be a semi-simple Lie group acting isometrically on a finite volume pseudo-riemannian manifold $M$. Let $Y$ be a nilpotent element of the Lie algebra $\mathscr{G}$ (that is ad $d_{Y}$ is a nilpotent endomorphism of $\mathscr{G}$ ). Then its orbits are (affinely parameterized) geodesics.

Proof. This follows from the fact that in a semi-simple Lie algebra $\mathscr{G}$, an element $Y$ is nilpotent exactly if it is normalized as above by some element $X$, that is $[X, Y]=-Y$. Indeed it is known that if $Y$ is nilpotent, then it belongs to some subalgebra isomorphic to $\operatorname{sl}(2, \mathbf{R})$.

\subsection{First and second fundamental forms for Killing algebras}

Let $G$ be a Lie group acting isometrically on $(M,<,>)$, and let $\mathscr{G}$ be its Lie algebra. The previous situation suggests that one can define some symmetric bilinear forms that describe the geometry of orbits. They will in fact be defined on $\mathscr{G}$ instead of the standard ones, which are defined on the orbits.

Definition 4.2 For $x \in M$, we call the following symmetric forms on $\mathscr{G}$, respectively first and second fundamental form:

$$
\begin{gathered}
I_{x}: \mathscr{G} \times \mathscr{G} \rightarrow \mathbf{R}, I_{x}(X, Y)=<X(x), Y(x)> \\
I I_{x}: \mathscr{G} \times \mathscr{G} \rightarrow T_{x} M, I I_{x}(X, Y)=-(1 / 2)[X, Y](x)+\nabla_{X} Y(x)
\end{gathered}
$$

Remark 4.3 Of course, the second fundamental form may be defined for affine actions. However, there is no means to generalize the first fundamental form.

With the help of these notions one may express previous results in terms of the vanishing of the second fundamental form $I I$. Observe that this is stronger than the fact that the orbits are geodesic: II is a kind of parametrized second fundamental. Its vanishing implies that the induced connection comes from the canonical one on the group. As an example, we can consider the (transitive) action of $S L(2, \mathbf{R})$ on a Hopf torus $\mathbf{R}^{2}-\{0\} / x \rightarrow \lambda x$. It has non vanishing second fundamental form. However we shall later $(\S 9)$ see that there is an equivalence between being geodesic and the fact $I I=0$, for actions of semi-simple groups preserving a connection and a volume. 


\subsection{Split Lie groups}

Corollary 4.4 (of 4.1) Let $G$ be a semi-simple Lie group acting isometrically on a compact pseudo-riemannian manifold $(M,<,>)$. Then the first and second fundamental forms vanish on the nilpotent cone of $\mathscr{G}$ (that is the set of nilpotent elements of $\mathscr{G}$ )

So, we are going to ask what are the (scalar or vectorial) forms on a semisimple Lie algebra which vanish on its nilpotent cone. One can easily see that bi-invariant forms (e.g. the Killing form) satisfy this property. More precisely:

Question 4.5 For what semi-simple Lie algebras, is the "quadratic envelope" of the nilpotent cone exactly the (Killing) light cone? (That is when is a quadratic form vanishing on the nilpotent cone a multiple of the Killing form?).

It is rather surprising that this question seems to have no classical known answer. Here we are not interested in a characterization of such algebras, but only in finding a substantial class of them.

Split semi-simple Lie algebras are those (real) Lie algebras having the same structure as the complex Lie algebras, that is they have systems of roots satisfying the same list of properties as for complex semi-simple Lie algebras. By definition a split Lie algebra has a (real) split rank, equal to the (usual) rank of its complexified algebra [Bou]. This implies many nice properties:

Proposition 4.6 Let $\mathscr{G}$ be a split simple Lie algebra (e.g. $\mathscr{G}=s l(n, \mathbf{R}), n \geq 2)$. Let $E$ be a vector space and $q: \mathscr{G} \times \mathscr{G} \rightarrow E$ a vector symmetric bilinear form, which vanishes on the nilpotent cone of $\mathscr{G}$. Then there is a unique $N \in E$ such that $q=\kappa N$, where $\kappa$ is the Killing form of $\mathscr{G}$.

Proof. We firstly observe that the vectorial case follows from the scalar one. Indeed write $q=\Sigma q_{i} e_{i}$ for some basis $\left\{e_{i}\right\}$ of $E$. Then each $q_{i}$ satisfies the hypothesis, that is $q_{i}=0$ on the nilpotent cone, and hence $q_{i}=a_{i} \kappa$ for some $a_{i}$. Thus $q=\kappa\left(\Sigma a_{i} e_{i}\right)$.

To prove the scalar case we argue by contradiction. Assume there is a space $\mathscr{E}_{0}$ of dimension $>1$ of such forms. Thus $\mathscr{G}$ acts on $\mathscr{E}_{0}$, with exactly the line $\mathbf{R} \kappa$ determined by the Killing form $\kappa$, as a set of fixed elements. Let $\mathscr{E}$ be an invariant supplementary of $\mathbf{R} \kappa$. Let $\mathscr{B}$ be a Borel subalgebra of $\mathscr{S}$, i.e. a maximal solvable Lie subalgebra, and $\mathscr{C}$ the Cartan subalgebra of $\mathscr{G}$ contained in $\mathscr{B}$.

Then there is an order $>$ on $R$ such $\mathscr{B}=\mathscr{b} \oplus \cdot \mathscr{N}^{+}$and $\mathscr{G}=\mathscr{b} \oplus \mathscr{N}^{+}$ $\bigoplus \mathscr{N}^{-}$, where: $\mathscr{N}^{+}=\sum_{\alpha>0} \mathscr{G}^{\alpha}$ and $\mathscr{N}^{-}=\bigoplus \sum_{\alpha>0} \mathscr{G}^{-\alpha}$. Here $\mathscr{G}^{\alpha}$ is the eigenspace associated to the root $\alpha$. (It is the split property of $\mathscr{G}$ that allows us to not add in the last sum, a $\mathscr{G}^{0}$ factor, which may be non trivial in the general case).

Standard facts from representation theory of semi-simple Lie groups imply that there is a non trivial element $q \in \mathscr{E}$, such that $\mathbf{R} q$ is left invariant under $B$. More precisely: $X_{\alpha} q=0$ for $\alpha>0, X_{\alpha} \in \mathscr{G}^{\alpha}$, and there is a homomorphism 
$\rho: \mathscr{A} \rightarrow \mathbf{R}$, such that $H q=\rho(H) q$ for $H \in \mathscr{C}$. This implies: $(\alpha(H)+$ $\beta(H)) q\left(X_{\alpha}, X_{\beta}\right)=\rho(H) q\left(X_{\alpha}, X_{\beta}\right)$, for any $H \in \mathscr{l}, X_{\alpha} \in \mathscr{G}^{\alpha}$ and $X_{\beta} \in \mathscr{G}^{\beta}$. In particular if there is $X_{\alpha}$ and $X_{\beta}$ such that $q\left(X_{\alpha}, X_{\beta}\right) \neq 0$, then $\alpha+\beta=\rho$ (in particular $\rho$ belongs to $R$ ).

Now, we apply our hypothesis, that is $q$ vanishes on the nilpotent cone. Observe that each $\mathscr{G}^{\alpha}$ consists of nilpotent elements. In fact, also the sum $\mathscr{G}^{\alpha} \bigoplus \mathscr{G}^{\beta}$, for $\alpha \neq-\beta$, consists of nilpotent elements. Indeed, in that case there is $H \in \mathscr{C}$, such that $\alpha(H)=\beta(H) \neq 0$ and hence $\operatorname{ad}(H)\left(X_{\alpha}+X_{\beta}\right)=$ $\alpha(H)\left(X_{\alpha}+X_{\beta}\right)$, for $X_{\alpha} \in \mathscr{G}^{\alpha}$ and $X_{\beta} \in \mathscr{G}^{\beta}$. The last property that $X_{\alpha}+X_{\beta}$ satisfies, is known to characterize nilpotent elements.

Therefore $q\left(X_{\alpha}, X_{\beta}\right)=0$ unless $\alpha=-\beta$. However, from above, in order that $q\left(X_{\alpha}, X_{-\alpha}\right) \neq 0$, we must have: $\rho=0$. However, this means that $\mathscr{B}$ fixes $q$. But this implies that $\mathscr{G}$ itself fixes $q$. (This is a standard fact of representation theory, that it suffices to verify for $s l(2, \mathbf{R}))$. Hence $q$ is a multiple of the Killing form, which contradicts our choice.

Thus for any $\alpha$ and $\beta, \mathscr{G}^{\alpha}$ is orthogonal to $\mathscr{G}^{\beta}$.

By the same argument we prove that $\mathscr{C}$ is orthogonal to itself and to all the $\mathscr{G}^{\alpha}$, except, probably, $\mathscr{G}^{\rho}$. For this last space, we apply to $q\left(X_{-\alpha}, X_{\rho}\right)$, the fact that $q$ is $a d\left(X_{\alpha}\right)$ antisymmetric when $\alpha>0$. Thus: $q\left(\left[X_{\alpha}, X_{-\alpha}\right], X_{\rho}\right)=$ $-q\left(X_{-\alpha},\left[X_{\alpha}, X_{\rho}\right]\right)$. The element $H_{\alpha}=\left[X_{\alpha}, X_{-\alpha}\right]$ belongs to $\mathscr{b}$ and $\left[X_{\alpha}, X_{\rho}\right]$ is nilpotent for $\alpha \neq-\rho$. Hence the previous orthogonalities yield: $q\left(H_{\alpha}, X_{\rho}\right)=0$ if $\alpha \neq-\rho$. Finally, the orthogonality $q\left(H_{\rho}, X_{\rho}\right)=0$, may be obtained by directly proving the vanishing of the restriction of $q$ to the subalgbra, isomorphic to $\operatorname{sl}(2, \mathbf{R}), s_{\rho}=\mathbf{R} H_{\rho} \bigoplus \mathscr{G}^{\rho} \bigoplus \mathscr{G}^{-\rho}$. Thus we have proved that $q=0$, which contradicts our hypotheses.

I would like to thank Y. Benoist who clarified me on some points concerning these notions.

\subsection{Geometrical implications}

Theorem 4.7 Let $G$ be a simple split Lie group acting isometrically on a finite volume pseudo-riemannian manifold $M$. Then the first and second fundamental forms are bi-invariant. That is for any $x \in M$, there is $\alpha(x) \in \mathbf{R}$ and $N(x) \in T_{x} M$ such that $I_{x}=\alpha(x) \kappa$ and $I I_{x}=\kappa N(x)$, where $\kappa$ is the Killing form of $\mathscr{G}$.

Theorem 4.8 (Umbilical equation) Let $G$ be a simple split Lie group acting isometrically on a finite volume pseudo-riemannian manifold $(M,<,>)$. Let $\kappa$ be the Killing form on the Lie algebra $\mathscr{G}$. Then there is a vector field (the principal normal field) $N$ on $M$ as regular as the data such that, for all Killing fields $X$ an $Y$ of $\mathscr{G}$, and all $x$ in $M$, we have:

$$
\nabla_{X} Y(x)=(1 / 2)[X, Y](x)+\kappa(X, Y) N(x)
$$

(that is $I I=\kappa N)$. 
Proof. From 4.6, for $x \in M$, there is unique $N(x) \in T_{x} M$ such that $I I_{x}=\kappa N(x)$. If $X \in \mathscr{G}$ satisfies $\kappa(X, X)=1$, then $N(x)=I I_{x}(X(x), X(x))$. This proves the regularity of $N$.

The next result relates the first and second fundamental forms. It follows from the previous theorem and 2.3

Theorem 4.9 (Unified equation) Let $G$ be a simple split Lie group acting isometrically on a finite volume pseudo-riemannian manifold $(M,<,>)$. Let $\kappa$ be the Killing form on the Lie algebra $\mathscr{G}$. Then there is a function $\alpha$ on $M$ as regular as the data, determining the first and second fundamental forms by: $I_{x}=\alpha(x) \kappa$ and $I I_{x}=\kappa \operatorname{grad}_{x} \alpha$. That is, for all Killing fields $X$ and $Y$ of $\mathscr{G}$, and all $x$ in $M$, we have:

$$
\nabla_{X} Y(x)=(1 / 2)[X, Y](x)+\kappa(X, Y) \operatorname{grad}_{x} \alpha
$$

Example 4.10 Let $G / \Gamma$ be a compact quotient of $G$ ( $\Gamma$ is discrete) and let $\left(V, g_{V}\right)$ be compact riemannian manifold. Consider on the product $G / \Gamma \times V$ the warped metric $g_{(x, v)}=\alpha(v) \kappa \bigoplus g_{v}$, where $\alpha$ is a function on $V$. The unified equation above is obvious, where in fact $\operatorname{grad} \alpha$ stands for the usual riemannian gradient.

\section{Local freedom of isometric pseudo-riemannian actions}

Here we deal with the question by [D-G]

Question 5.1 Is a faithful smooth volume preserving action of a semi-simple Lie group $G$ with non compact factor, on a compact manifold $M$, everywhere locally free?

A motivation of the question is a classical measure theoretic result of Zimmer stating that this is true almost everywhere, in fact without any smoothness or compactness condition.

One may expect in the presence of these conditions uniform local freedom since this is the case for all known (those given earlier) examples.

We have already proved (everywhere) local freedom of pseudo-riemannian actions of semi-simple Lie groups, in the ergodic (3.2) and isotropic (3.1) cases. This was also proved by [Gro], in the case of actions of semi-simple Lie groups acting isometrically on compact pseudo-riemannian manifolds, of signature $(p, q)$, such that $\operatorname{dim}(G)>\min (p, q)$. Other cases are treated in [A-S] and [Zeg] (see also [S-Z] for related results).

We present in what follows our third, and perhaps the most important, local freedom result. Later another one will be discussed concerning affine actions (§8).

Theorem 5.2 Let $G$ be a simple split Lie group acting isometrically on a finite volume pseudo-riemannian manifold $M$. Suppose that $G$ is not locally isomorphic to $S L(2, \mathbf{R})$. Then the action of $G$ is everywhere locally free. 
(The exception of $\operatorname{SL}(2, \mathbf{R})$ is due to the method of proof, and has no profound significance).

Proof. The proof is algebraic using algebraic information on the stabilizer algebra, derived from the umbilical equation 4.8. We argue by contradiction, so consider for $x \in M$, the stabilizer algebra $\mathscr{H}=\{X \in \mathscr{G} / X(x)=0\}$, and suppose this is non trivial.

Let us first show that: $\mathscr{H} \neq \mathscr{G}$, that is $x$ is not a fixed point of $G$. From 4.3, every $\kappa$-lightlike element $X$ of $\mathscr{G}$, has (lightlike) geodesic orbits. Therefore, if $X$ is singular at $x$, from $8.2,\left(D_{x} X\right)^{2}=0$. Thus in particular, the infinitesimal representation of $G$ on $T_{x} M$, maps lightlike elements to nilpotent elements of $\operatorname{End}\left(T_{x} M\right)$, and hence these lightlike elements are themselves nilpotent. But this occurs only if $\mathscr{G}=\operatorname{sl}(2, \mathbf{R})$.

For $X \in \mathscr{H}$ and $Y \in \mathscr{G}, \nabla_{X} Y(x)=0$. Therefore, the umbilical equation 4.8 implies: $[X, Y](x)+\kappa(X, Y) N(x)=0$, for any $Y \in \mathscr{G}$. Since there is $Y$, such that $\kappa(X, Y) \neq 0, N(x)$ is tangent to the orbit $G x$, at $x$. Let $\tilde{N}$ be an element of $\mathscr{G}$ projecting onto $N(x)(\tilde{N}$ is defined modulo $\mathscr{H})$.

Fact 5.3 $\mathscr{H}$ satisfies the " almost ideal property": $X \in \mathscr{H}, Y \in \mathscr{G} \Longrightarrow$ $[X, Y] \in \mathscr{H} \bigoplus \mathbf{R} \tilde{N}$. Moreover $\mathscr{H}$ is $\kappa$ isotropic

To see that $\mathscr{H}$ is isotropic, suppose the contrary: for some $X \in \mathscr{H}, \kappa(X, X) \neq 0$. This implies $N(x)=0$, that is $\tilde{N} \in \mathscr{H}$, i.e. $\mathscr{H}$ is an ideal.

From the fact we deduce that $\mathscr{H}$ is solvable. Indeed if $\mathscr{H}$ contains a semisimple subalgebra, this then contains non trivial elements which are diagonalizable over $\mathbf{R}$. By definition of $\kappa$ such elements are not isotropic.

Therefore $\mathscr{H}$ is contained in a Borel subalgebra $\mathscr{B}$ of $\mathscr{G}$, i.e. a maximal solvable Lie subalgebra. Let $\mathscr{C}$ be the Cartan subalgebra of $\mathscr{B}$ and $R$ be the associated root system. Then $\mathscr{G}=\mathscr{b} \oplus \sum_{\alpha \in R} \mathscr{G}^{\alpha}$, where $\mathscr{G}^{\alpha}$ is the eigenspace associated to the root $\alpha$. Let $S$ be a simple system of roots, inducing an order $>$ on $R$. Then: $\mathscr{B}=\mathscr{b} \oplus \sum_{\alpha>0} \mathscr{G}^{\alpha}$ and: $\mathscr{G}=\mathscr{b} \oplus \sum_{\alpha>0} \mathscr{G}^{\alpha} \bigoplus \sum_{\alpha>0} \mathscr{G}^{-\alpha}$. Since it is $\kappa$ isotropic, $\mathscr{H}$ must be contained in $\mathscr{N}=\sum_{\alpha>0} \mathscr{G}^{\alpha}$. This last nilpotent algebra lies in the kernel of the restriction of $\kappa$ to $\mathscr{B}$. But from the umbilical equation, $X \in \mathscr{H}, Y \in \mathscr{G}, \kappa(X, Y)=0 \Longrightarrow[X, Y] \in \mathscr{H}$. This implies in particular that $\mathscr{H}$ is an ideal of $\mathscr{B}$. Therefore we have a splitting: $\mathscr{B}=\sum_{\alpha>0} \mathscr{B} \cap \mathscr{G}^{\alpha}$. Since $\operatorname{dim}\left(\mathscr{G}^{\alpha}\right)=1$ (because $\mathscr{G}$ is split), $\mathscr{H}$ must contain some $\mathscr{G}^{\alpha}$. But $\left[\mathscr{G}^{\alpha}, \mathscr{G}^{-\alpha}\right]=\mathscr{b}^{\alpha}$ is a 1-dimensional vector subspace of $\mathscr{l}$. Thus, from the Fact above, one may choose $\tilde{N}$ to be a generator of $\mathscr{C}^{\alpha}$.

Suppose that $\mathscr{H}$ contains another $\mathscr{G}^{\beta}$ (of course $\beta>0$ ). Then applying the same argument yields: $\left[\mathscr{S}^{\beta}, \mathscr{S}^{-\beta}\right]=\left[\mathscr{G}^{\alpha}, \mathscr{S}^{-\alpha}\right]$. However, it is known that in general, for a root $\gamma$, some generator of $\left[\mathscr{G}^{\gamma}, \mathscr{G}^{-\gamma}\right]$ is the dual of the root $\gamma$. Hence, the last equality implies $\alpha$ and $\beta$ are proportional. This is impossible.

Now the above fact translates to: $\left[\mathscr{G}, \mathscr{G}^{-\alpha}\right] \subset \mathscr{G}^{-\alpha} \bigoplus \cdot \mathscr{C}^{\alpha}$. But for $\beta \neq$ $-\alpha$, if $\left[\mathscr{S}^{-\alpha}, \mathscr{G}^{\beta}\right] \neq 0$, then $\alpha+\beta$ is a root and $\left[\mathscr{S}^{-\alpha}, \mathscr{G}^{\beta}\right]=\mathscr{S}^{\alpha+\beta}$. Obviously, this last eigenspace does not intersect $\mathscr{G}^{\alpha} \bigoplus \mathscr{C}^{\alpha}$. Therefore, we must have: $\left[\mathscr{G}^{-\alpha}, \mathscr{G}^{\beta}\right]=0$, for any $\beta \neq \alpha$. In the same way, $\left[\mathscr{G}^{\alpha}, \mathscr{G}^{\beta}\right]=0$, since the 
structure is invariant under the canonical involution sending any $\mathscr{G}^{\gamma}$ to $\mathscr{S}^{-\gamma}$. Therefore the subalgebra $\mathscr{S}^{\alpha}=\mathscr{G}^{\alpha} \bigoplus \mathscr{G}^{-\alpha} \bigoplus \cdot \mathscr{C}^{\alpha}$ (isomorphic to $\operatorname{sl}(2, \mathbf{R})$ is an ideal of $\mathscr{G}$. This contradicts the hypotheses of the Theorem, which is thus completely proved.

Remark 5.4 (A project of proof using the gradient of $\alpha$ ). From the unified equation 4.9 , one sees that a singular orbit of a point $x$ is isotropic $(\alpha(x)=0)$ and geodesic $\left(\operatorname{grad}_{x} \alpha\right.$ is tangent to the orbit), but $\operatorname{grad}_{x} \alpha \neq 0$, unless $x$ is a fixed point of $G$ (one may analyse the case of fixed point separately). Therefore near $x$, the level $\alpha^{-1}(0)$ is regular (i.e. is a hypersurface) and $\operatorname{grad} \alpha$ leaves it invariant. This situation does not happen for gradients taken in the sense of a riemannian metric, but always occurs in symplectic geometry. In our hypothetic present situation, we see how pseudo-riemannian geometry mixes riemannian and symplectic aspects.

\section{On an embedding theorem of Zimmer}

If a finite volume manifold $M=G / \Gamma$ admits a $G$-invariant pseudo riemannian metric then the quadratic form $b$ on $\mathscr{G}$ that it represents is $A d(\Gamma)$ invariant. If $G$ is semi-simple with no compact factor, then by the Borel density theorem, $b$ will be in fact $A d(G)$ invariant. Thus in particular $A d(G)$ embeds in the orthogonal group of $b$.

R. Zimmer [Zim1] generalizes this fact to not necessarily transitive actions but which just preserve measure and some $H$-structure [Zim]. His proof was ergodic theoretic. M. Gromov then proposes a geometric proof by making $\operatorname{Ad}(G)$ act locally isometrically.

Here we propose an elementary proof (of course without using previous results but rather similar simpler ideas) in the case (in fact the most useful) of pseudo-riemannian structures and actions of split Lie groups. So we assume $G$ acts isometrically on a manifold $M$ endowed with a pseudo-riemannian metric of type $(p, q)$. We then prove that $\mathscr{G}$ embeds in $o(p, q)$, the Lie algebra of the orthogonal group of a standard form of signature $(p, q)$ on $\mathbf{R}^{p+q}$.

\subsection{The non isotropic case}

Fix a point $x$ in $M$ and identify $T_{x} M$ with $\mathbf{R}^{p+q}$. Suppose that the orbit $G x$ is not isotropic: $I_{x}$ is not identically 0 . If $G$ is a semi-simple split Lie group, then from 4.7, $I_{x}=\alpha \kappa$ for some $\alpha \neq 0$. It follows in particular that $G$ acts locally freely on $G x$ (we assume that $\mathscr{G}$ is simple). We may then identify $T_{x}(G x)$ with $\mathscr{G}$. Therefore $\mathscr{G}$ endowed with its Killing form is isometrically embedded as a non degenerate subspace in $\mathbf{R}^{p+q}$. Thus $\operatorname{Ad}(G)$ embeds in $o(p, q)$, acting as usual on $\mathscr{G}$, and trivially on its orthogonal. 


\subsection{The totally isotropic case}

Now we suppose that $G$ is Lie group (not necessarily semi-simple and split) acting isometrically on a pseudo-riemannian manifold $M^{p, q}$ of finite volume, with all the orbits of $G$ isotropic.

We fix $x$ and identify $T_{x} M$ with $\mathbf{R}^{p, q}$. We denote as previously $D_{x} X: T_{x} M \rightarrow$ $T_{x} M$ the covariant derivative of $X$ at $x$. By $2.1, X$ is a Killing field exactly when $D_{x} X$ is antisymmetric, that is $D_{x} X$ belongs to the orthogonal Lie algebra $o(p, q)$. However, when an algebra $\mathscr{G}$ of Killing fields acts, the collection of $D_{x} X$ for $X \in \mathscr{G}$ is by no means a subalgebra of $o(p, q)$, isomorphic to $\mathscr{G}$ (see for example [K-N] for the homomorphic defect of the correspondence $X \in \mathscr{G} \rightarrow$ $\left.D_{x} X \in o(p, q)\right)$.

The proof of the next proposition which implies the embedding theorem under the isotropic hypothesis, follows immediately from 3.1.

Proposition 6.1 Let G be a Lie group acting isometrically on a pseudo-riemannian manifold $M$ with isotropic orbits. Let $x$ be a point of $M$ and identify as above $T_{x} M$ with $\mathbf{R}^{p+q}$ and the restriction $D_{x} X / T_{x}(G x)$ with $(1 / 2) a d_{X}$ (acting on $\mathscr{G}$ ). Let $t$ be the subalgebra of $o(p, q)$ generated (as a subalgebra) by the vector subspace $\left\{D_{x} X, X \in \mathscr{G}\right\}$ of $o(p, q)$. Then the restriction map $D_{x} X \in \mathscr{b} \rightarrow$ $2 D_{x} X / T_{x}(G x)=a d_{X} \in \operatorname{ad}(\mathscr{G})$ extends to an onto homomorphism $\mathscr{b} \rightarrow \operatorname{ad}(\mathscr{G})$. In particular, if $\mathscr{G}$ is simple, it embeds in $\mathscr{C} \subset o(p, q)$.

Example 6.2 The maximal dimension of an isotropic space of a pseudo-riemannian metric of type $(p, q)$ is $\min (p, q)$. Therefore if $\operatorname{dim}(G)>\min (p, q)$ (and $G$ is simple to ensure local freedom from 3.1), then $G$ can not act (even locally) with isotropic orbits. This is sharp. Let us give a homogeneous example of an isometric action of $S L(2, \mathbf{R})$ with isotropic orbits where the metric is of type $(3,3)$. Take $I$ to be $S L(2, \mathbf{C})$ and $M=S L(2, \mathbf{C}) / \Gamma$ where $\Gamma$ is a co-compact lattice. We endow the Lie algebra $s l(2, \mathbf{C})$ with a bi-invariant form (different from the Killing form) $b$ for which $s l(2, \mathbf{R})$ is isotropic. We just take $b$ to be the imaginary part of the $\mathbf{C}$-Killing form on $s l(2, \mathbf{C})$. (The Lie algebra of $\operatorname{sl}(2, \mathbf{C})$ is identified with the space of complex $2 \times 2$ matrices $A$, with $\operatorname{tr}(A)=0$. Then $b(A, B)=\operatorname{tr}(\sqrt{-1} A B))$.

\section{Affine actions. The associated affine envelope}

(One may consult [Fer] and [Goe] for recent works on affine actions). Let $(M, \nabla)$ be an affine manifold (this means that $\nabla$ is a torsion free connection on $M$, not necessarily flat). Let $\chi(M)$ be the space of smooth vector fields on $M$. So $\nabla$ is a bilinear map $\chi(M) \times \chi(M) \rightarrow \chi(M)$. For $\mathscr{B}$ a subspace of $\chi(M)$ let $\nabla(\mathscr{B})$ be the subspace of $\chi(M)$ generated by $\nabla(\mathscr{B} \times \mathscr{B})$. That is $\nabla(\mathscr{B})$ is the vector space generated by the vector fields $\nabla_{X} Y$ for $X, Y \in \chi(M)$. We define $\nabla^{k}(\mathscr{B})=\nabla^{k-1}(\nabla(\mathscr{B}))$ inductively.

Observe that, due to the torsion free property: $[X, Y]=\nabla_{X} Y-\nabla_{Y} X$, we have $[\mathscr{B}, \mathscr{B}] \subset \nabla(\mathscr{B})$. 
Definition 7.1 We call $\mathscr{B}^{\nabla}=\sum_{k \in \mathbf{N}} \nabla^{k}(\mathscr{C})$ the affine envelope of $\mathscr{B}$. It is a Lie subalgebra of $\chi(M)$ (generally of infinite dimension), invariant under the operation $\nabla$. Its orbits are (totally) geodesic submanifolds of $M$.

Let $G$ be a Lie group acting isometrically on $(M, \nabla)$. Then $\mathscr{G}$ may be seen as a subspace of $\chi(M)$. Since the action of $G$ is connection preserving, $G$ acts naturally on the (finite dimensional) spaces $\nabla^{k}(\mathscr{G})$, and acts on the algebra $\mathscr{G} \nabla$, respecting the operation $\nabla$. Observe that (for example), if $G$ is perfect $([\mathscr{G}, \mathscr{G}]=\mathscr{G})$, then $\mathscr{G} \subset \nabla(\mathscr{S})$, and in particular, $\mathscr{G} \nabla$ is an extension of $\mathscr{G}$.

Example 7.2 Consider $M=\mathbf{R}^{n}$ endowed with the linear action of $G=S L(n, \mathbf{R})$. Then the affine envelope extension is just $G L(n, \mathbf{R})$. The operation $\nabla$ on the Lie algebra $g l(n, \mathbf{R})$ is the usual multiplication of matrices.

Let now $\mathscr{S}$ be reduced to a linear vector field $A \in M_{n \times n}$. Then $\mathscr{S}^{\nabla}$ is the vector subspace of $M_{n \times n}$ generated by the (integer) powers of $A:\left\{A, A^{2}, \ldots, A^{k}, \ldots\right\}$.

In general $\mathscr{G}^{\nabla}$ is the associative subalgebra of $M_{n \times n}$ generated by $\mathscr{G}$.

Example 7.3 In the example $4.10, \mathscr{G}^{\nabla}$ is the direct sum of $\mathscr{G}$ with $(\mathbf{R} \operatorname{grad} \alpha)^{\nabla}$ (the last $\nabla$ is the Levi-Civita connection of the riemannian manifold $\left(V, g_{V}\right)$ ). In particular $\mathscr{S}$ may have infinite dimension.

Example 7.4 As in the situation of 2.2, let $X, Y$ be two vector fields on an affine manifold $(M, \nabla)$ such that $[X, Y]=-Y$. Suppose that $X$ is Killing field, preserving a finite smooth measure. Consider $\mathscr{N}=(\mathbf{R} Y)^{\nabla}$, the affine envelope of $\mathbf{R} Y$. Then $\mathscr{N}$ is a nilpotent finite dimensional Lie algebra, having a one parameter group of homotheties induced by the action of $X$. All of these facts are straightforward (for example, to prove that $\mathscr{N}$ has finite dimension, observe that, if it is not trivial, $\nabla_{Y} Y$ belongs to the -2 stable Liapunov space of $X$, and so on...).

Theorem 7.5 Let $G$ be a semi-simple Lie group without compact factor acting isometrically on an affine unimodular (smooth) manifold $(M, \nabla, \omega)$, of finite volume. Suppose the action is ergodic. Then the affine envelope extension $G^{\nabla}$ preserves the volume form and is a finite dimensional Lie algebra.

Proof. We first show that $G^{\nabla}$ is volume preserving. Consider $\nabla^{k}(\mathscr{S})$, and let $E$ be the finite dimensional space of functions on $M$, of the form $f=\operatorname{div}_{\omega} X$ for $X \in \nabla^{k}(\mathscr{G})$ (recall that the divergence $\operatorname{div} X$ is defined by $L_{X} \omega=\operatorname{div} X \omega$, where $L_{X}$ is the Lie derivative). Then $G$ acts on $E$, respecting the $L^{2}$ hilbertian scalar product $\langle f, g\rangle=\int_{M} f g \omega$. Since $G$ is semi-simple with no compact factor, $G$ acts trivially on $E$ since $E$ is finite dimensional. That is the elements of $E$, are $G$ invariant functions on $M$, and hence are constant by ergodicity. But a divergence function $\operatorname{div} X$, has vanishing mean: $\int_{M} \operatorname{div} X=0$. Therefore the vector fields of $\nabla^{k}(\mathscr{G})$ are volume preserving.

Denote $\mathscr{G}^{k}=\nabla^{k}(\mathscr{G})$ and for $x \in M$, let $\mathscr{G}^{k}(x)$ be the evaluation of $\mathscr{G}^{k}$ at $x: \mathscr{G}^{k}(x)=\left\{X(x) \in T_{x} M / X \in \mathscr{G}^{k}\right\}$. Let $k$ be the smallest integer such that $\sum_{i \leq k} \mathscr{G}^{i}(x)=\mathscr{G}^{\nabla}(x)$, for $x$ in a subset of $M$ of positive measure (and hence 
full measure by ergodicity). We show that $\sum_{i \leq k} \mathscr{G}^{i}=\mathscr{G}^{\nabla}$. Let $\mathscr{B}(x) \in \mathscr{G}^{k+1}$ be the kernel of the evaluation of $\mathscr{G}^{k+1}$ at $x: \mathscr{B}(x)=\left\{X \in \mathscr{G}^{k+1} / X(x)=\right.$ $0\}$. By ergodicity, the dimension of $\mathscr{D}(x)$ is almost everywhere a constant $d$. So we have a Gauss map: $x \in M \rightarrow \mathscr{B}_{x} \in G r^{d}\left(\mathscr{G}^{k+1}\right)$. It is an equivariant map where $G$ acts naturally on $G r^{d}\left(\mathscr{G}^{k+1}\right)$. A standard argument (using the Furstenberg lemma [Zim2]), because of the ergodicity, shows that the Gauss map is constant. That is there is subspace $\mathscr{B} \subset \mathscr{G}^{k+1}$, such that for almost all $x$, $\mathscr{B}(x)=\mathscr{B}$. By definition of $k$, for any $X \in \mathscr{G}^{k+1}$, there is $Y \in \sum_{i \leq k} \mathscr{G}^{i}$, such that $X(x)-Y(x)=0$, that is $X-Y \in \mathscr{B}(x)$. By constancy of $\mathscr{B}, X-Y=0$ almost everywhere. Thus $X \in \sum_{i \leq k} \mathscr{G}^{i}$.

\subsection{Affine structure of the orbits of $G^{\nabla}$}

An orbit $G^{\nabla} x$ is identified with the quotient $G^{\nabla} / H_{x}$. As a geodesic submanifold of $M$, it possesses a connection. By definition of $\mathscr{G} \nabla$, the covariant derivation determined by this connection preserves $\mathscr{G} \nabla$. This means that $\nabla$ lifts to a right invariant connection on $G$. As in 1.2.1, this is a warped connection on $\mathscr{G} \nabla$ given by a symmetric tensor $W: \mathscr{G} \nabla \times \mathscr{G} \nabla \rightarrow \mathscr{G} \nabla$. The fact that the left action of $G$ preserves the connection means that: $W$ is $\operatorname{Ad}(G)$-invariant.

Fact 7.6 Let $d \pi_{x}: \mathscr{G}^{\nabla} \rightarrow T_{x}\left(G^{\nabla} x\right) \subset T_{x} M$ be the derivative at the neutral element of $G^{\nabla}$ of the projection $G^{\nabla} \rightarrow G^{\nabla} x$. Then the second fundamental form of $G$ is given by $I_{x}(X, Y)=d \pi_{x} W(X, Y)$. In particular it is equivariant with respect to some representation of $G$ in $T_{x} M$.

Remark 7.7 It is very restrictive for a right invariant connection on a group $G^{\nabla}$, to be projectable as a connection on a quotient $G^{\nabla} / H$, for $H$ non discrete. This gives further evidences for the local freedom question, perhaps, even for $G^{\nabla}$.

\section{Toward local freedom}

\subsection{Singularities of vector fields}

Let $(M, \nabla)$ be a affine manifold. Let $x$ be a fixed point of $M$. A vector field $X$ (not necessarily Killing) singular at $x$, i.e. $X(x)=0$, has a well defined derivative, defined in charts, which is an endomorphism of $T_{x} M$. In fact it just equals the covariant derivative $D_{x} X$ (at $x$ ). This does not depend upon the connection $\nabla$. Indeed it may be defined canonically using the flow generated by $X$, or just by $D_{x} X(Z)=-[X, Z]$ for $Z$ a vector field.

Lemma 8.1 Let $X$ and $Y$ be two vector fields singular at $x$. Then $D_{x}\left(\nabla_{X} Y\right)=$ $D_{x} Y D_{x} X$.

Proof. Let $R$ be the curvature tensor at $x$, then $R(X, Z) Y=0$ for any vector field $Z$. Thus $0=R(X, Z) Y=\nabla_{X} \nabla_{Z} Y-\nabla_{Z} \nabla_{X} Y-\nabla_{[X, Z]} Y$. Then we apply the various previous equivalent definitions of $D_{x} X$ and $D_{x} Y$. 
Corollary 8.2 Let $X$ be a vector field vanishing at $x$. Suppose that $X$ has (affinely parameterized) geodesic orbits. Then $\left(D_{x} X\right)^{2}=0$.

Proof. We apply the previous lemma since our condition means: $\nabla_{X} X=0$.

Corollary 8.3 ([A-S]) A Killing vector field of a Lorentzian metric with geodesic orbits, has no singularities.

Proof. Let $A$ be an element of an orthogonal algebra $o(p, q)$ then for any $u \in$ $\mathbf{R}^{p, q}, A u$ is orthogonal to $u$. If furthermore $A^{2}=0$, then the image $\operatorname{Im}(A)$ is isotropic. Therefore, $\operatorname{Im}(A)$ can not have dimension 1 , since otherwise it would be orthogonal to the whole space $\mathbf{R}^{p, q}$. Thus in the lorentzian case, $A=0$.

Remark 8.4 (Heuristic approach) The previous facts are trivial in the flat case (i.e. on $\mathbf{R}^{n}$ ), where furthermore the vector fields are linear. The general case may be roughly treated using infinitesimal flatness of connections. More precisely, let $x \in M$ and $\exp _{x}: U \subset T_{x} M \rightarrow M$ be the exponential map defined in a neighborhood $U$ of 0 in $T_{x} M$. On $U$, let $\nabla^{\lambda}$ be the connection pull back of $\nabla$ via the map $f^{\lambda}: u \in U \rightarrow \exp _{x}(\lambda u) \in M$. Then $\nabla^{\lambda}$ converges (in the smooth topology) to the flat connection on $T_{x} M$, when $\lambda \rightarrow 0$.

\subsection{The stabilizer subalgebras of $\mathscr{G} \nabla$}

The following fact appears very interesting (for applications):

Theorem 8.5 Let $G$ be as in 7.5, that is $G$ is a semi-simple Lie group without compact factor, that acts isometrically and ergodically on an affine unimodular manifold $M$ of finite volume. Then for $x \in M$, the infinitesimal representation of its stabilizer subalgebra $\mathscr{G} \nabla(x)$ in $T_{x} M$ is nilpotent. In particular $\mathscr{G}(x)=\mathscr{G} \cap$ $\mathscr{G}^{\nabla}(x)$ is (as an abstract group) nilpotent (since this last algebra is represented faithfully in $T_{x} M$, because it preserves a connection).

Proof. Let $\rho: \mathscr{G}^{\nabla}(x) \rightarrow \operatorname{End}\left(T_{x} M\right)$, be the infinitesimal representation, $\rho(X)=$ $D_{x} X$. But $\mathscr{G}^{\nabla}(x)$ is $\nabla$-invariant. This implies, from 8.1 that the image $\rho\left(\mathscr{G}^{\nabla}(x)\right)$ is (an associative) subalgebra of $\operatorname{End}\left(T_{x} M\right)$. So, if $A$ belongs to $\rho\left(\mathscr{G}^{\nabla}(x)\right)$, then this is also the case of each power $A^{2}, \ldots, A^{k}, \ldots$ Now the volume conservation property of elements of $G^{\nabla}$, yields that for such an element $A, \operatorname{trace}\left(A^{k}\right)=0$, for any integer $k$. This implies that $A$ is nilpotent.

Example 8.6 Let $G=S l(2, \mathbf{R})$ acting (transitively) affinely on a Hopf torus. Then $G^{\nabla}=G L(2, \mathbf{R})$. The infinitesimal representation of the stabilizer of any point, is equivalent to the standard representation of the affine group, but its restriction to $S l(2, \mathbf{R})$ is nilpotent.

\subsection{Reduction to the $S L(2, \mathbf{R})$ case}

From a result of $[\mathrm{Stu}]$, one may deduce: 
Fact 8.7 If a semi-simple Lie group with no compact factor $G$ acts, but not locally freely on a compact manifold, then some nilpotent one parameter group does not act locally freely. It then follows that some subgroup $G^{\prime}$ locally isomorphic to $S L(2, \mathbf{R})$ does not act locally freely (in fact for such a subgroup, all nilpotent one parameter groups are somewhere singular) .

Let us assume, to simplify, that $G^{\prime}$ is indeed (globally) isomorphic to $S L(2, \mathbf{R})$. So questions on local freedom may be reduced to that of actions of $\operatorname{SL}(2, \mathbf{R})$, which is therefore, the most difficult case. Let us study in more details this case.

We use the classical (dynamical) generators $X, Y$ and $Z$ for the Lie algebra $\operatorname{sl}(2, \mathbf{R}): X=\left(\begin{array}{cc}-1 / 2 & 0 \\ 0 & 1 / 2\end{array}\right)$ is hyperbolic, $Y=\left(\begin{array}{ll}0 & 1 \\ 0 & 0\end{array}\right)$ and $Z=\left(\begin{array}{ll}0 & 0 \\ 1 & 0\end{array}\right)$ are nilpotent. We have the relations: $[X, Y]=-Y,[X, Z]=Z$ and $[Y, Z]-2 X$.

\subsubsection{Stabilizer subalgebras}

Fact 8.8 Let $S L(2, \mathbf{R}) \subset G$, and $G$ acts ergodically on an affine unimodular manifold $M$ of finite volume (as in 7.5). Then:

(i) $\operatorname{Sl}(2, \mathbf{R})$ has neither fixed points nor one dimensional orbits.

(ii) The isotropy algebra of a singular orbit is a nilpotent one dimensional algebra, i.e. conjugate to $\mathbf{R} Y$ (or equivalently to $\mathbf{R} Z$ ).

Proof. (i) In this case, the isotropy algebra will be a nilpotent subalgbra (8.5) of dimension 2 or 3 in $\operatorname{sl}(2, \mathbf{R})$. This is impossible.

(ii) From 8.5 , if $A$ belongs to an isotropy algebra, then $a d_{A}$ is always a nilpotent endomorphism of $s l(2, \mathbf{R}) / \mathbf{R} A$. This implies that $A$ is nilpotent.

\subsubsection{A proof of local freedom, modulo a technical condition}

From above, a singular orbit is identified as a homogeneous space to $\mathbf{R}^{2}-\{0\}$ or to a Hopf torus $\mathbf{R}^{2}-\{0\} /\{x \rightarrow \lambda x\}$, endowed with the $S L(2, \mathbf{R})$ linear action. So, the idea is to consider the induced geometric structure on such an orbit, and if all things work naturally, then use the (8.6), that is, the restricted stabilizer subalgebra is not nilpotent. Unfortunately, this presupposes, that there is a way to induce the connection on the orbits. This is of course the case if they are geodesic, but we were not able to prove that, although we are convinced that it is the case. So we have a weaker result:

Theorem 8.9 Let $G$ be a semi-simple Lie group with no compact factor acting isometrically and ergodically on an affine unimodular manifold $(M, \nabla, \omega)$ of $f$ nite volume. Then $\left(S L(2, \mathbf{R}), \mathbf{R}^{2}-\{0\}\right)$ can not be immersed equivariantly and geodesically in $M$, with image contained in an orbit of $G$.

Proof. We argue by contradiction: suppose that for some $x, Y(x)=0(Y \in$ $\operatorname{sl}(2, \mathbf{R})$ is as above). Then $T=\nabla_{Y} Z$ is also singular at $x$. So, by $8.5, D_{X} T$ 
is nilpotent. However, our geodesibility hypothesis implies that $T$ preserves the $S L(2, \mathbf{R})$ orbit of $x$, and that the calculus of the restriction of $D_{x} T$ to the tangent space of that orbit, is the same as in the model $\mathbf{R}^{2}-\{0\}$. But there, a computation gives, for any $x, D_{x} T=D_{x}\left(\nabla_{Y} Z\right)=Z Y=\left(\begin{array}{ll}0 & 0 \\ 0 & 1\end{array}\right)$. This is not nilpotent.

\section{Further remarks}

\subsection{Ergodic theory for the second fundamental form}

Our philosophy here was to use direct concrete (and sometimes constructive) methods. These methods are not always sufficiently powerful to obtain the most general statements. They provide however a guide to the formulation of a more general theorems. For example, using the Zimmer's machinery, we can prove (without giving details here) the following generalization of facts that we have already proved, for some of them only for split semi-simple groups, and for others only in the pseudo-riemannian case, or for ergodic actions.

Theorem 9.1 Let $G$ be a semi-simple Lie group with no compact factor acting isometrically on an affine unimodular manifold $(M, \nabla, \omega)$ of finite volume. Then:

(i) For any $x \in M$, the second fundamental form $I_{x}: \mathscr{G} \times \mathscr{G} \rightarrow T_{x} M$ is equivariant with respect to some representation of $\mathscr{G}$ in $T_{x} M$.

(ii) $I I_{x}=0$ everywhere, if and only if the orbits are geodesic. In that case, the action is everywhere locally free.

(iii) In the pseudo-riemannian case the first fundamental form is bi-invariant.

\subsection{Structure of the singular set of an $\operatorname{SL}(2, \mathbf{R})$ action}

Singular sets of group actions come with nice and rich structures that sometimes give evidence to their non existence. Let for example, $G=S L(2, \mathbf{R})$ act on a compact manifold $M$. Suppose that $G$ has no 0 or 1 -dimensional orbits, that is, every orbit $G x$ has dimension 2 or 3.

The subset $F$ of points $x$ with orbit $G x$ of dimension 2, and having as isotropy algebra $\mathscr{G}(x)$ a one dimensional nilpotent subalgebra of $\operatorname{sl}(2, \mathbf{R})$, is a closed non empty $G$-invariant subset. This subset has the structure of a 2-dimensional lamination, say $\mathscr{B}$, parameterized by the $G$ action.

It also has a tangential vector field $D$, commuting with the action (this is equivalent to say that the $S L(2, \mathbf{R})$ action extends to a $G L(2, \mathbf{R})$ action). Indeed a leaf is covered by $\mathbf{R}^{2}-\{0\}$, and the flow of $D$ induces multiplication $x \rightarrow e^{t} x$.

One way to see this is coherently defined in $F$, is the following. We use the notations $X, Y, Z \ldots$ in the previous section. Let $x \in F$ and consider the projection $\pi_{x}: G \rightarrow G x$ and $d_{1} \pi_{x}: \mathscr{G} \rightarrow T_{x}(G x)$. If the neutral component of the stabilizer $\mathscr{G}(x)$ is $A d(g)(\mathbf{R} Y)$, then take: $D(x)=d_{1} \pi_{x}(A d(g) X)$. One then cheeks that this does not depend upon the choice of $g$. 
Observe the following amusing fact about the the flow $\phi^{t}$ determined by $X$. A compact leaf of $\mathscr{B}$, is a Hopf torus $\mathbf{R}^{2}-\{0\} /\{x \rightarrow \lambda x\}$. The foliation determined by $X$ on such a torus is a Reeb foliation. It has no remarkable dynamic property, other than what one may call $C^{0}$ ergodicity. That is a continuous invariant function by a Reeb foliation, must be constant. This fact generalizes to $\left(F, \phi^{t}\right)$ (without assuming existence of compact leaves).

Fact 9.2 (Mautner property) A continuous function on $F$ that is invariant by $\phi^{t}$, is in fact $G$-invariant (i.e. constant on the leaves of $\mathscr{D}$ ).

Proof. Let $f$ be such a function. It is enough to prove that $f$ is invariant by the flows of $Y$ and $Z$. The argument is the same for both of these flows. Let us show it for the flow $\psi^{s}$ generated by $Y$. The identity $[X, Y]=-Y$ translates to the normalization relation: $\phi^{t} \psi^{s}=\psi^{s \exp -t}$. Fix $s$, and consider $f\left(\psi^{s} x\right)-f(x)$. By $\phi^{t}$ invariance of $f$, this equals $f\left(\phi^{t} \psi^{s} x\right)-f\left(\phi^{t} x\right)$, for any $t$. This equals $f\left(\psi^{s \exp -t} \phi^{t} x\right)-f\left(\phi^{t} x\right)=f\left(\psi^{s \exp -t} y\right)-f(y)$, where $y=\phi^{t} x$. The last oscillation tends uniformly to 0 when $t \rightarrow \infty$, since $\psi^{s \exp -t} \rightarrow$ Identity.

\section{References}

[A-S] S. Adams, G. Stuck: The isometry group of a compact Lorentz manifold, Invent. Math. 129 (1997) 239-261.

[Bour] N. Bourbaki: Groupes et algèbres de LIe, ch 7, 8, CCLS Paris (1995).

[D-G] G. D'Ambra and M. Gromov: Lectures on transformation groups: geometry and dynamics, Surveys in Differential Geometry (Supplement to the Journal of Differential Geometry) 1 (1991) 19-111.

[Fer] R. Feres: Actions of discrete linear groups and Zimmer's conjecture, J. Diff. Geom. 42 (1995) 554-576.

[Goe] E. Goetze: Connection preserving actions of connected and discrete Lie groups, J. Diff. Geom. 40 (1994), 595-620.

[Gro] M. Gromov: Rigid transformation groups, Géometrie différentielle. D. Bernard et ChoquetBruhat. Ed. Travaux encours 33. Paris. Hermann (1988).

[K-N] S. Kobayashi, K. Nomizu: Foundations of differential geometry, volume 1, Interscience, New York, 1963.

[M-R] A. Medina, Ph. Revoy: Algèbres de Lie et produit scalaire invariant, Ann. Scien. Éc. Norm. Sup. 18 (1985), 553-561.

[Stu] G. Stuck: Low dimensional actions of semisimple Lie groups, Is. Jour. Math 76 (1991), 27-71.

[S-Z] G. Stuck, R. Zimmer Stabilizers for ergodic actions of higher rank semisimple groups Ann. Math 139 (1994), 723-747.

[Zeg] A. Zeghib: Sur les espaces-temps homogènes, preprint (1995).

[Zim1] R. Zimmer: On the automorphism group of a compact Lorentz manifold and other geometric manifolds, Invent. Math. 83 (1986) 411-426.

[Zim2] R. Zimmer: Ergodic theory and semisimple Lie groups, Birkhäuser, 1984, Boston. 